
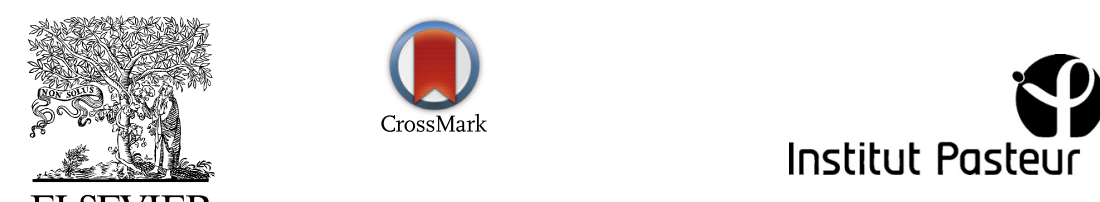

\title{
Development and optimization of a new MALDI-TOF protocol for identification of the Sporothrix species complex
}

\author{
Manoel Marques Evangelista Oliveira ${ }^{\mathrm{a}, \mathrm{b}, \mathrm{c}, *}$, Cledir Santos ${ }^{\mathrm{b}, \mathrm{d}}$, Paula Sampaio ${ }^{\mathrm{c}}$, Orazio Romeo ${ }^{\mathrm{e}}$, \\ Rodrigo Almeida-Paes ${ }^{a}$, Célia Pais ${ }^{c}$, Nelson Lima ${ }^{\text {b,d }}$, Rosely Maria Zancopé-Oliveira ${ }^{\text {a }}$

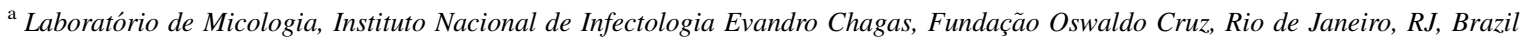 \\ ${ }^{\mathrm{b}}$ CEB-Centre of Biological Engineering, Micoteca da Universidade do Minho, Universidade do Minho, Campus de Gualtar, Braga, Portugal \\ ${ }^{\mathrm{c}}$ Centre of Molecular and Environmental Biology (CBMA), Department of Biology, Universidade do Minho, Braga, Portugal \\ d Programa de Pós-Graduação em Microbiologia Agrícola, Universidade Federal de Lavras, Lavras, MG, Brazil \\ ${ }^{\mathrm{e}}$ Department of Environmental and Biological Sciences, University of Messina, Messina, Italy
}

Received 7 September 2014; accepted 15 December 2014

Available online 3 January 2015

\begin{abstract}
Accurate species identification of the Sporothrix schenckii complex is essential, since identification based only on phenotypic characteristics is often inconclusive due to phenotypic variability within the species. We used matrix-assisted laser desorption ionization time-of-flight mass spectrometry (MALDI-TOF MS) for species identification of 70 environmental and clinical isolates of the Sporothrix complex. A reference database was established for MALDI-TOF MS-based species identification according to minor adjustments in the manufacturer's guidelines. The MALDI-TOF MS clearly distinguished strains of Sporothrix brasiliensis, Sporothrix globosa, Sporothrix mexicana, S. schenckii, Sporothrix luriei and Sporothrix pallida, enabling identification of all isolates at the species level, as confirmed by partial calmodulin gene sequence analyses. The present methodology is simple, reliable, rapid and highly suitable for routine identification in clinical mycology laboratories and culture collections, particularly for updating and reclassifying of deposited Sporothrix isolates.
\end{abstract}

(C) 2014 Institut Pasteur. Published by Elsevier Masson SAS. All rights reserved.

Keywords: Sporothrix complex; Sporotrichosis; MALDI-TOF MS; Calmodulin gene

\section{Introduction}

Sporotrichosis is a globally distributed subcutaneous mycosis, with areas of high endemicity in Latin America, South Africa, India, China and Japan [1,2]. It is usually associated with puncture injuries in farmers, florists, leisure gardeners, nursery workers, landscapers and greenhouse workers [3]. Moreover, in several Brazilian states, especially in Rio de Janeiro, a hyperendemic region of sporotrichosis,

\footnotetext{
* Corresponding author. Setor de Imunodiagnóstico, Laboratório de Micologia do Instituto Nacional de Infectologia Evandro Chagas, Fundação Oswaldo Cruz, Av. Brasil 4365, Manguinhos, Rio de Janeiro 21040-900, Brazil. Tel.: +55 213865 9640; fax: +55 2138659557 .

E-mail address: manoel.marques@ini.fiocruz.br (M.M.E. Oliveira).
}

transmission of the disease has been widely occurring in the populace via scratches and bites of cats naturally infected with Sporothrix spp. [3].

The diagnosis of sporotrichosis is classically attained by correlation of clinical, epidemiological and laboratory data, including culture and fungal phenotypic characteristics. Although studies in the past few years have considered Sporothrix schenckii as a single taxon, Liu et al. [4] reported the existence of genetic variation within isolates belonging to this species. In addition, other studies using different methodologies, such as M13 PCR fingerprinting, have demonstrated that $S$. schenckii isolates have different genetic characteristics, which suggests that they do not belong to the same species $[1,5]$.

Through the association of phenotypic traits and sequencing data, Marimon et al. [6] recognized three new 
species, Sporothrix brasiliensis, Sporothrix globosa and Sporothrix mexicana, and proposed an identification key for the Sporothrix species complex. In addition to molecular data, species identification included conidial morphology, growth rates and auxonogram analysis using raffinose and sucrose as carbon sources. S. globosa has been defined as having worldwide distribution [1,7]. On the other hand, S. brasiliensis was believed to be restricted to Brazil $[6,8,9]$ and $S$. mexicana to environmental samples from Mexico [6]. Recently, however, S. mexicana was found in a human patient in Portugal [10] and in re-identification of three clinical isolates maintained in fungal collections since 1955 [9]. Taxonomic revision of S. schenckii var. luriei as a new species, Sporothrix luriei, was also proposed [11]. Furthermore, phylogenetic analysis based on the rDNA and $\beta$-tubulin regions from Sporothrix albicans, Sporothrix pallida and Sporothrix nivea revealed significant similarity, with the proposal of designating all of these species as $S$. pallida [12]. Based on the latter study, Romeo and collaborators studied the molecular phylogeny and epidemiology of $S$. schenckii complex strains isolated in Italy, and demonstrated that 26 environmental strains co-clustered with $S$. pallida and two clinical strains grouped with $S$. schenckii stricto sensu [13]. Nonetheless, a recent report showed that $S$. pallida caused keratitis in a corneal transplant recipient [14].

The correlation between molecular data and phenotypic characteristics was described as fundamental to the identification of species of the Sporothrix complex [8]. Therefore, in recent years, taxonomists are seeking tools for rapid and reliable identification of this complex $[15,16]$. Nevertheless, research on the identification of species belonging to the Sporothrix complex has been neglected in the literature. Matrix-assisted laser-desorption/ionization time-of-flight mass spectrometry (MALDI-TOF MS) is an example of an emerging tool for microorganism characterization and differentiation at the species and, in some cases, strain level [17-20].

MALDI-TOF MS methodology requires a relatively small number $\left(10^{6}-10^{7}\right)$ of microbial cells [21]. It has been applied in the field of mycology in order to generate a spectrum that is characteristic of each fungal taxon. Its remarkable reproducibility is based on measurement of proteins in a mass range between 2000 and 20,000 Da, where important ribosomal proteins could be used as biomarkers. The entire process is reviewed and described in detail for analysis of yeasts $[19,22]$ and filamentous fungi $[20,23]$. At present, several important contributions have been published on MALDI-TOF MS for fungal identification [24-31]. However, there is a paucity of studies applying this technique to thermally dimorphic fungi such as Histoplasma capsulatum, Coccidioides posadasii, Paracoccidioides brasiliensis or the Sporothrix complex. In addition, reference spectra for these dimorphic fungi are scarce in commercially available databases.

The aim of this study was to develop and optimize a new MALDI-TOF protocol for the identification of clinical and environmental isolates of the Sporothrix species complex based on their proteomic profiles, by direct analysis of fungal colonies. Furthermore, construction and validation of a reference database, with development of a specific database for routine identification of the $S$. schenckii complex, was achieved. Our findings emphasize that MALDI-TOF MS is a reliable, fast and accurate method for routine identification of the Sporothrix complex.

\section{Materials and methods}

\subsection{Strains, phenotypic identification and growth conditions}

This study was carried out with 70 Sporothrix isolates (Table 1) previously characterized as $S$. schenckii by classical morphology [12] and divided into two groups of strains. The first group, used to build the reference database for the MALDI-TOF MS (Axima LNR system, Kratos Analytical, Shimadzu, UK), consisted of 6 reference strains belonging to the following clinically and environmentally relevant Sporothrix species: S. brasiliensis (CBS120339/IPEC16490), $S$. schenckii (IPEC27722), S. globosa (IPEC27135), S. pallida (SPA8), S. mexicana (MUM11.02) and S. luriei (CBS937.72) (Table 1). The second group, that included 64 clinical and environmental isolates obtained from different culture collections, was used to evaluate the new Sporothrix complex MALDI-TOF MS database engineered in this study.

Fungi were subcultured on Sabouraud dextrose agar and mycobiotic agar (both from Difco ${ }^{\mathrm{TM}}$ BD/Sparks, MD, USA) and then presumptively identified at the species level by phenotypic characteristics (macro and micromorphology, thermotolerance and carbohydrate assimilation) as previously described [8,32]. A standardized method was established for microbial growth for MALDI-TOF MS analysis. Each Sporothrix isolate was subcultured on the yeast-like form using brain-heart infusion (BHI) agar or yeast extract peptone dextrose (YEPD, peptone $2 \%$, yeast extract $1 \%$, dextrose $2 \%$ ) and on the filamentous form using potato dextrose agar (PDA). Cultures were incubated for a period ranging from 3 to 8 days at $25,34,35.5$ or $37^{\circ} \mathrm{C}$. Escherichia coli strain DH5 $\alpha$ was obtained from the Portuguese Fungal Culture Collection, Micoteca da Universidade do Minho, MUM (WDCM816 and ISO 9001:2008 Certification $N^{\circ}$ : PT-2011/CEP.3911) and it was used for in situ extraction of proteins, which were used as standard for MALDI-TOF MS external calibration. E. coli DH5 $\alpha$ cells were grown on Luria-Bertani medium agar $(10 \mathrm{~g} /$ $\mathrm{L}$ tryptone, $5 \mathrm{~g} / \mathrm{L}$ yeast extract, $10 \mathrm{~g} / \mathrm{L} \mathrm{NaCl}$ ) at $37^{\circ} \mathrm{C}$ for $20 \mathrm{~h}$ as previously described $[17,19]$.

\subsection{Molecular analysis}

As the gold standard method for species designation in the Sporothrix complex, genomic DNA was obtained from the fungal mycelial phase and sequencing of the partial calmodulin (CAL) gene was performed at the sequencing platform of the Fundação Oswaldo Cruz (PDTIS/FIOCRUZ), Rio de Janeiro (Brazil) as previously described [8]. Sequences from both DNA strands were generated and edited 
Table 1

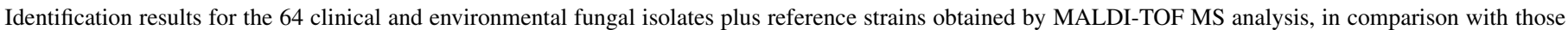
obtained by sequencing of the partial calmodulin gene and phenotypic tests.

\begin{tabular}{|c|c|c|c|c|c|}
\hline \multirow[t]{2}{*}{ Strain } & \multirow[t]{2}{*}{ Phenotypic identification $^{\mathrm{d}}$} & \multicolumn{3}{|c|}{ Genotypic characterization } & \multirow{2}{*}{$\frac{\text { Proteomic characterization }}{\text { MALDI-TOF MS }}$} \\
\hline & & Final identification $^{\mathrm{b}}$ & Genbank no & References $^{c}$ & \\
\hline IPEC 16490 & S. brasiliensis & S. brasiliensis & AM116899 & 6 & S. brasiliensis \\
\hline IPEC27445-3 & S. brasiliensis & S. brasiliensis & HQ426950 & 8 & S. brasiliensis \\
\hline IPEC27052 & Sporothrix spp. ${ }^{a}$ & S. brasiliensis & HQ426941 & 8 & S. brasiliensis \\
\hline IPEC27135 & Sporothrix spp. ${ }^{\mathrm{a}}$ & S. globosa & GU456632 & 32 & S. globosa \\
\hline IPEC27387 & Sporothrix spp. ${ }^{\mathrm{a}}$ & S. brasiliensis & HQ426948 & 8 & S. brasiliensis \\
\hline IPEC34067 & Sporothrix spp. ${ }^{\mathrm{a}}$ & S. brasiliensis & HQ426952 & 8 & S. brasiliensis \\
\hline IPEC27372 & Sporothrix spp. ${ }^{\mathrm{a}}$ & S. brasiliensis & HQ426947 & 8 & S. brasiliensis \\
\hline IPEC25011 & S. brasiliensis & S. brasiliensis & HQ426935 & 8 & S. brasiliensis \\
\hline IPEC33605 & Sporothrix spp. ${ }^{a}$ & S. brasiliensis & HQ426957 & 8 & S. brasiliensis \\
\hline IPEC27930 & Sporothrix spp. ${ }^{\mathrm{a}}$ & S. brasiliensis & HQ426951 & 8 & S. brasiliensis \\
\hline IPEC28772 & Sporothrix spp. ${ }^{a}$ & S. brasiliensis & HQ426955 & 8 & S. brasiliensis \\
\hline IPEC34007 & Sporothrix spp. ${ }^{a}$ & S. brasiliensis & HQ426959 & 8 & S. brasiliensis \\
\hline IPEC27177-2 & Sporothrix spp. ${ }^{\mathrm{a}}$ & S. brasiliensis & HQ426944 & 8 & S. brasiliensis \\
\hline IPEC27288 & Sporothrix spp. ${ }^{a}$ & S. brasiliensis & HQ426945 & 8 & S. brasiliensis \\
\hline IPEC27209 & Sporothrix spp. ${ }^{\mathrm{a}}$ & S. brasiliensis & HQ426946 & 8 & S. brasiliensis \\
\hline IPEC28604 & S. brasiliensis & S. brasiliensis & HQ426953 & 8 & S. brasiliensis \\
\hline IPEC26945 & Sporothrix spp. ${ }^{\mathrm{a}}$ & S. brasiliensis & HQ426939 & 8 & S. brasiliensis \\
\hline IPEC27130 & Sporothrix spp. ${ }^{\text {a }}$ & S. brasiliensis & HQ426943 & 8 & S. brasiliensis \\
\hline IPEC16919 & S. brasiliensis & S. brasiliensis & HQ426930 & 8 & S. brasiliensis \\
\hline IPEC18782A & S. brasiliensis & S. brasiliensis & HQ426933 & 8 & S. brasiliensis \\
\hline IPEC28329 & S. schenckii & S. brasiliensis & JN995610 & 15 & S. brasiliensis \\
\hline IPEC27022 & S. brasiliensis & S. brasiliensis & HQ426940 & 8 & S. brasiliensis \\
\hline IPEC28665 & S. brasiliensis & S. brasiliensis & JN995606 & 15 & S. brasiliensis \\
\hline IPEC29334 & S. schenckii & S. schenckii & HQ426962 & 8 & S. schenckii \\
\hline IPEC26961 & S. schenckii & S. schenckii & JN995605 & 15 & S. schenckii \\
\hline IPEC27100 & S. schenckii ${ }^{\mathrm{a}}$ & S. brasiliensis & JN995609 & 15 & S. brasiliensis \\
\hline IPEC27133 & S. schenckii ${ }^{\mathrm{a}}$ & S. brasiliensis & JN995608 & 15 & S. brasiliensis \\
\hline MUM 11.02 & S. mexicana or $S$. schenckii ${ }^{\mathrm{a}}$ & S. mexicana & JF970258 & 10 & S. mexicana \\
\hline IPEC17692 & Sporothrix spp. ${ }^{a}$ & S. brasiliensis & HQ426929 & 8 & S. brasiliensis \\
\hline IPEC17521 & S. brasiliensis ${ }^{\mathrm{a}}$ & S. schenckii & KC463901 & This study & S. schenckii \\
\hline IPEC30650 & S. brasiliensis & S. brasiliensis & KC463893 & This study & S. brasiliensis \\
\hline ATCC32285 & S. schenckii & S. schenckii & KJ472131 & This study & S. schenckii \\
\hline INSA378027 & Sporothrix spp. ${ }^{\mathrm{a}}$ & S. globosa & KC716083 & 1 & S. globosa \\
\hline MEL14 & Sporothrix spp. ${ }^{\mathrm{a}}$ & S. schenckii & KJ472133 & This study & S. schenckii \\
\hline IPEC25374 & S. brasiliensis & S. brasiliensis & KC463894 & This study & S. brasiliensis \\
\hline IPEC17585 & S. schenckii & S. schenckii & KC463902 & This study & S. schenckii \\
\hline ATCC32286 & S. schenckii & S. schenckii & KJ472132 & This study & S. schenckii \\
\hline IPEC27722 & S. mexicana ${ }^{\mathrm{a}}$ & S. schenckii & HQ426961 & 8 & S. schenckii \\
\hline BG6 & S. pallida & S. pallida & HQ692915 & 13 & S. pallida \\
\hline EH194 & S. schenckii & S. schenckii & KJ472123 & This study & S. schenckii \\
\hline CBS937.72 & S. luriei & S. luriei & AM747302 & 11 & S. luriei \\
\hline KMU975 & S. schenckii & S. schenckii & KJ472142 & This study & S. schenckii \\
\hline SPA1 & S. pallida & S. pallida & KJ472126 & This study & S. pallida \\
\hline SPA8 & S. pallida & S. pallida & HQ686039 & 13 & S. pallida \\
\hline SAM1 & S. pallida & S. pallida & KJ472130 & This study & S. pallida \\
\hline BG2 & S. pallida & S. pallida & KJ472128 & This study & S. pallida \\
\hline BG & S. pallida & S. pallida & KJ472127 & This study & S. pallida \\
\hline SPA2 & S. pallida & S. pallida & KJ472129 & This study & S. pallida \\
\hline 12013-08 & S. schenckii & S. schenckii & KJ472141 & This study & S. schenckii \\
\hline $01022-10$ & S. schenckii & S. schenckii & KJ472139 & This study & S. schenckii \\
\hline IPEC29333 & S. schenckii & S. schenckii & HQ426960 & 8 & S. schenckii \\
\hline IPEC27375 & S. schenckii & S. brasiliensis & KC463898 & This study & S. brasiliensis \\
\hline $14879-07$ & Sporothrix spp. ${ }^{a}$ & S. globosa & KJ472125 & This study & S. globosa \\
\hline IPEC27558-1 & S. schenckii ${ }^{\mathrm{a}}$ & S. brasiliensis & KC463899 & This study & S. brasiliensis \\
\hline IPEC25758 & S. brasiliensis & S. brasiliensis & KC463895 & This study & S. brasiliensis \\
\hline $10036-08$ & S. schenckii & S. schenckii & KJ472138 & This study & S. schenckii \\
\hline IPEC27454 & S. brasiliensis & S. brasiliensis & KC463896 & This study & S. brasiliensis \\
\hline IPEC17608 & Sporothrix spp. ${ }^{\mathrm{a}}$ & S. brasiliensis & AM116890 & 6 & S. brasiliensis \\
\hline IPEC17307 & S. schenckii ${ }^{\mathrm{a}}$ & S. brasiliensis & KC463892 & This study & S. brasiliensis \\
\hline 03017-07 & S. schenckii & S. schenckii & KJ472134 & This study & S. schenckii \\
\hline
\end{tabular}


Table 1 (continued)

\begin{tabular}{|c|c|c|c|c|c|}
\hline \multirow[t]{2}{*}{ Strain } & \multirow[t]{2}{*}{ Phenotypic identification $^{\mathrm{d}}$} & \multicolumn{3}{|c|}{ Genotypic characterization } & \multirow{2}{*}{$\begin{array}{l}\text { Proteomic characterization } \\
\text { MALDI-TOF MS }\end{array}$} \\
\hline & & Final identification $^{b}$ & Genbank no & References $^{\mathrm{c}}$ & \\
\hline IPEC 17920 & S. brasiliensis & S. brasiliensis & AM116888 & 6 & S. brasiliensis \\
\hline IPEC27466 & S. schenckii ${ }^{\mathrm{a}}$ & S. brasiliensis & KJ482637 & This study & S. brasiliensis \\
\hline IPEC25853 & S. brasiliensis & S. brasiliensis & KJ482636 & This study & S. brasiliensis \\
\hline IPEC25303 & S. schenckii ${ }^{\mathrm{a}}$ & S. brasiliensis & KC463891 & This study & S. brasiliensis \\
\hline IPEC25457 & S. brasiliensis & S. brasiliensis & KC463890 & This study & S. brasiliensis \\
\hline 03022-06 & S. schenckii & S. schenckii & KJ472135 & This study & S. schenckii \\
\hline 11029-06 & S. schenckii & S. schenckii & KJ472140 & This study & S. schenckii \\
\hline IPEC24372-1 & S. schenckii & S. schenckii & KC463903 & This study & S. schenckii \\
\hline 03003-07 & S. schenckii & S. schenckii & KJ472136 & This study & S. schenckii \\
\hline 04015-07 & S. schenckii & S. schenckii & KJ472137 & This study & S. schenckii \\
\hline
\end{tabular}

\footnotetext{
${ }^{a}$ Incorrect phenotypic identification.

b Calmodulin sequencing.

c Reference of partial gene calmodulin sequencing.

d Reference of phenotypic identification 8 .
}

with the Sequencher 4.6 software package (Gene Codes Corporation, USA), followed by alignment by Mega version 4.0.2 software. Species identification was performed by searching databases (GenBank) with BLAST (Basic Local Alignment Search Tool, NIH). For phylogenetic tree construction, a bootstrap test with 1000 replicates was conducted for both Neighbor-joining and maximum parsimony analyses [8]. The isolate was assigned to a species if it had at least $98 \%$ homology with sequences present in the searched database. All CAL sequences from isolates included in genotypic analysis were deposited in the GenBank database under accession numbers shown in Table 1.

\subsection{MALDI-TOF MS}

\subsubsection{Sample preparation}

Briefly, to engineer the database, a set of reference isolates for each species were chosen and subcultured as described above. We transferred $10^{6}$ cells of each sample (c.a. $1 \mu \mathrm{g}$ ) from the culture plate to a $500 \mu \mathrm{L}$ tube containing $20 \mu \mathrm{L}$ of $25 \%$ formic acid in water (v/v). Samples were vortexed for $20 \mathrm{~s}$ and then sonicated at room temperature for $15 \mathrm{~min}$ and incubated at $4{ }^{\circ} \mathrm{C}$ for $3 \mathrm{~h}$. Supernatant of each sample $(1 \mu \mathrm{L})$ was transferred to a paraffin film surface and $2 \mu \mathrm{L}$ of a matrix solution were added and gently mixed. Two different matrices were evaluated in this study, 2,5-dihydroxybenzoic acid (DHB, Fluka, Buchs, Switzerland) $75 \mathrm{mg} / \mathrm{mL}$ in ethanol/ water/acetonitrile $(1: 1: 1)$ containing $0.03 \%(\mathrm{v} / \mathrm{v})$ trifluoroacetic acid [TFA, Fluka, Buchs, Switzerland] and $\alpha$-cyano-4hydroxycinnamic acid [CHCA, Fluka, Buchs, Switzerland) saturated in a solution with $33 \%$ ethanol, $33 \%$ acetonitrile, $31 \% \mathrm{H}_{2} \mathrm{O}$ and 3\% TFA. Each suspension $(1 \mu \mathrm{L})$ was spotted onto a MALDI-TOF MS stainless plate (FlexiMass ${ }^{\mathrm{TM}}$, Shimadzu Biotech, UK).

For the E. coli strain DH5 $\alpha$, about $10^{6}$ cells (c.a. $1 \mu \mathrm{g}$ ) were used. Cells were transferred into $500 \mu \mathrm{L}$ tubes containing $15 \mu \mathrm{L}$ of the matrix solution and then vortexed. Supernatant of each sample $(1 \mu \mathrm{L})$ was transferred to a paraffin film surface and an additional $2 \mu \mathrm{L}$ of matrix solution were added and gently mixed. Each suspension $(1 \mu \mathrm{L})$ was then spotted onto the MALDI-TOF MS stainless plate (FlexiMass ${ }^{\mathrm{TM}}$, Shimadzu Biotech, UK) in triplicate to test reproducibility. In case of discordant results, analysis was repeated for at least 2 additional replicates. Samples were air-dried at room temperature prior to spectra acquisition. During the sample preparation, all solutions were prepared and stored at $5{ }^{\circ} \mathrm{C}$.

\subsubsection{Spectra acquisition and statistical analysis}

Spectra acquisition was performed on Axima LNR equipment (Kratos Analytical, Shimadzu, UK) equipped with a nitrogen laser $(337 \mathrm{~nm})$. The laser intensity was set just above the threshold for ion production as previously described $[17,19]$. Twelve defined ribosomal proteins $(4365.40,5096.80$, $5381.40,6241.40,6255.40,6316.20,6411.60,6856.10$, $7158.80,7274.50,7872.10, \quad 9742.00$ and $12,227.30 \mathrm{Da})$ extracted in situ from the intact cells of E. coli strain DH5 $\alpha$ were used as external calibrants. A mass range from 2000 to $20,000 \mathrm{Da}$ was recorded using the MALDI-TOF MS linear mode with a delay of $104 \mathrm{~ns}$ and an acceleration voltage of $+20 \mathrm{kV}$.

For the DHB matrix, final spectra were generated by summing 20 laser shots accumulated per profile and 50 profiles produced per sample, leading to 1000 laser shots per summed spectrum. For the CHCA matrix, final spectra were generated by summing 2 laser shots accumulated per profile and 100 profiles produced per sample, leading to 200 laser shots per summed spectrum.

For data analysis, the resulting peak lists were exported into the SARAMIS ${ }^{\circledR}$ software package (Spectral Archiving and Microbial Identification System; AnagnosTec, Postdam-Golm, Germany) where final microbial identification was achieved. Peak lists of individual samples were compared to the SARAMIS $^{\mathrm{TM}}$ database, generating a ranked list of matching spectra. A minimum of 100 peaks and at least $98 \%$ mass similarity with the reference spectra of each strain were defined as acceptance criteria to validate results. Statistic grouping was obtained as described elsewhere [18]. Briefly, similarity within each spectrum was expressed as the relative or absolute number of matching mass signals after subjecting data to a single link agglomerative clustering algorithm. 


\section{Results}

\subsection{Molecular analysis}

Concerning molecular data, all isolates evaluated by CAL sequencing in this study were classified into the Sporothrix complex and distributed among the species $S$. brasiliensis $(n=38), S$. globosa $(n=3)$, S. pallida $(n=7)$, S. luriei $(n=1)$, S. mexicana $(n=1)$ and $S$. schenckii $(n=20)$ with high bootstrap support values (Fig. 1).

\subsection{Comparison of culture media and growth conditions}

A standardized method was established for microbial growth for MALDI-TOF MS analysis. Each Sporothrix isolate was subcultured in two culture media on the yeast-like form using BHI agar and YEPD, and on the filamentous form using PDA.

Preliminary MALDI-TOF MS analyses were performed daily for yeast cells incubated from 3 to 8 days at $34,35.5$ or $37^{\circ} \mathrm{C}$ and mycelial cells incubated from 3 to 14 days at $25^{\circ} \mathrm{C}$. Initial analyses for filamentous form showed only peaks with quality and without noise with 7-day-old cultures, but reproducible data were obtained only with 14-day-old colonies. Concerning BHI and YEPD media, optimal growth and quality of the spectra were obtained with BHI and, in contrast, no spectra or spectra of poor quality were frequently obtained from samples grown in YEPD medium.

\subsection{Reference database}

The first parameter studied with Sporothrix yeast cells was the influence of cell aggregation on the spectra. The established sample preparation based on pretreatment with a sonication bath appeared to minimize the aggregation of yeast cells, and therefore presented weaker interference of the cellular biomass concentration during material transfer to the target plate. Use of the established experimental procedure led to acquisition of reproducible spectra using the CHCA matrix (Fig. 2A). In contrast, when the sonication bath was not used, cellular aggregation in some isolates was observed. Sonication-free samples generated spectra with poor reproducibility (data not shown). The optimized cell number for spectral acquisition was $10^{6}$ cells per MALDI-TOF MS sample. The best results were obtained for samples prepared in the CHCA matrix with yeast cells grown between 3 and 6 days. During this period, no differences in spectral quality were found for any of the isolates evaluated using the CHCA matrix (Fig. 2A). Moreover, use of the CHCA matrix led to the acquisition of spectra with high peak gain (high number of peaks) up to the 8th day of fungal growth. In contrast, results obtained for the DHB matrix showed significant loss of peak gain over time after the 3rd day (Fig. 2B). From the 3rd to the 6 th day, peak resolution was also better when CHCA was used as the matrix (Fig. 3A and B).

For both CHCA and DHB matrices, methodologies of protein extraction led to different spectral results as a function of the matrix (Fig. 3A and B). When DHB was used as the matrix, spectral reproducibility was strongly affected, showing peaks with noise, loss of peaks, and consequently, a low level of reproducibility of the spectrum (Fig. 2B). In contrast, use of CHCA showed a high level of reproducibility over time (Fig. 2A).

During MALDI-TOF MS standardization, the influence of both culture media and temperature on spectral acquisition was also evaluated. Yeast growth in BHI agar between 3 and 6 days showed reproducible spectra with good quality. Therefore, Sporothrix yeast cells grown for 3 days in BHI agar were chosen for continuing our analyses. The standard deviation for each conserved peak did not exceed a $5 \mathrm{~m} / \mathrm{z}$ value (Fig. 4).

\subsection{Validation of the database}

In order to validate the database for identification of Sporothrix species, a group of 64 clinical and environmental isolates (37 S. brasiliensis, 19 S. schenckii, 2 S. globosa and 6 S. pallida) were used. Analysis of all isolates on MALDI-TOF MS demonstrated a high correlation with the reference spectra, ranging from 98 to $99 \%$ similarity. The methodologies used for identification of species of the Sporothrix complex were concordant and the standardized protocol for identification at the species level by MALDI-TOF MS permitted accurate analysis for all isolates (64/64) (Table 1).

\section{Discussion}

Differentiation of closely related Sporothrix species is a challenging and difficult issue in clinical mycology laboratories. Ribosomal proteins have been described as excellent candidates for microbe identification, as they are universal within cellular life [33]. Previous analyses with bacterial species reported that most ribosomal proteins are highly conserved, and some of these proteins are subject to slight variations at the strain level [34,35]. Therefore, variations in ribosomal protein genes have been proposed for classification and typing purposes in bacterial species through ribosomal multilocus sequence typing [36] or MALDI-TOF MS [37]. With the same rationale, the use of ribosomal protein mass analysis in a MALDI-TOF spectrum directly from fungal cells could provide an interesting tool for classification of Sporothrix isolates at the species level, as well as other proteins obtained by the extraction process used in the MALDI technology.

This study represents the first application of MALDI TOF MS technology for identifying clinical and environmental isolates of the Sporothrix complex, with generation of a library of protein masses representative of all six species described in this fungal complex.

In our work, better results were achieved with pretreated samples, as reported by Cassagne et al. [38] for other yeasts. These results are in agreement with previous studies $[39,40]$ demonstrating that, in some microorganisms such as yeasts, a prior protein extraction step may be necessary. Several methodologies, including exposing intact cells to high 


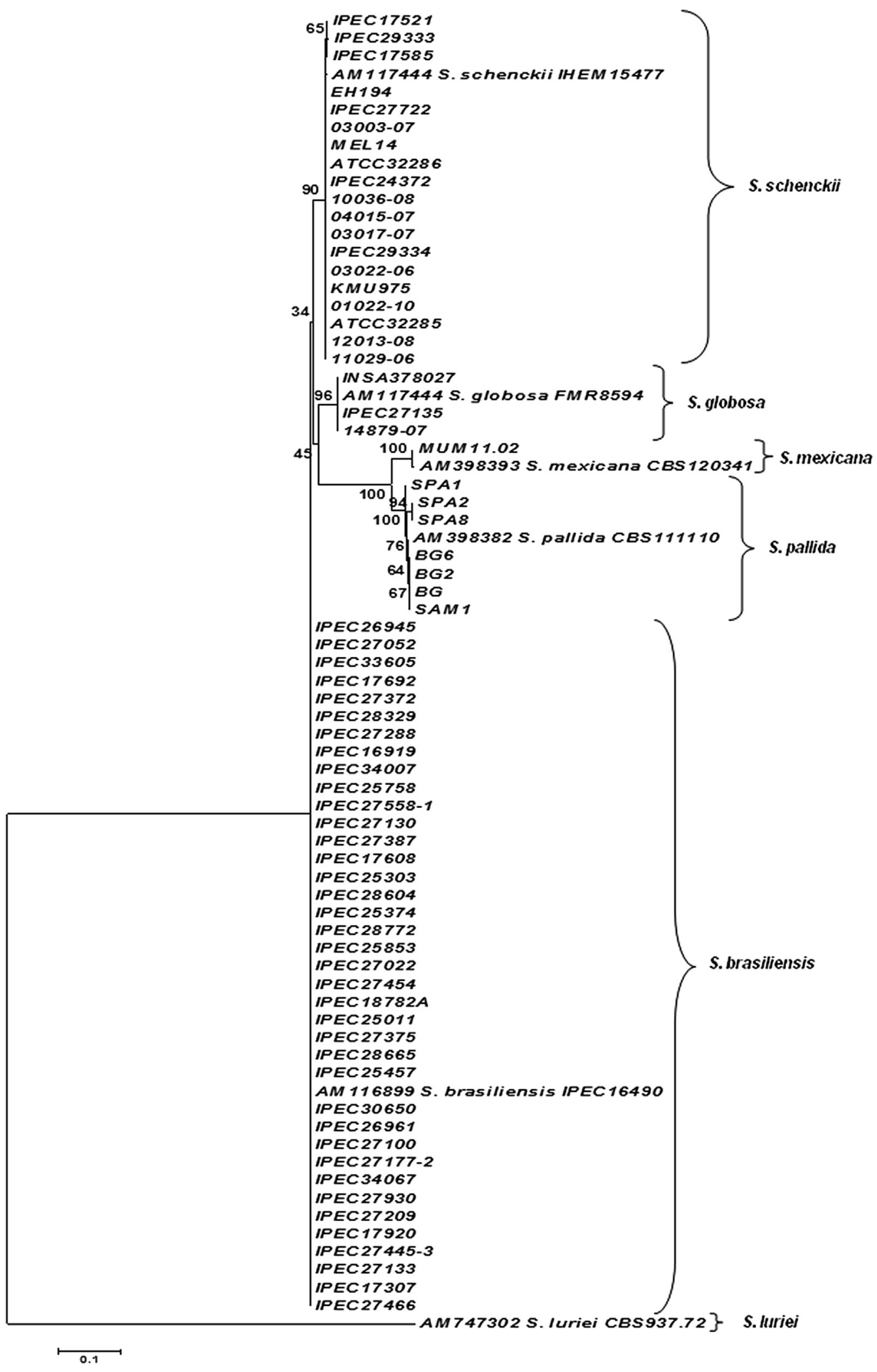

Fig. 1. Consensus tree of Sporothrix strains based on partial calmodulin (CAL) gene sequences and NCBI public GenBank sequences AM398393 (S. mexicana), AM398382 (S. pallida), AM116906 (S. globosa), AM116899 (S. brasiliensis), AM747302 (S. luriei) and AM117444 (S. schenckii). The tree was constructed with MEGA version 4.0.2 and 1000 bootstrap replicates. 
A

Shimadzu Biotech Axima LNR 28.6.20100624

\%olnt. $\quad 44 \mathrm{mV} 72 \mathrm{mV} 37 \mathrm{mV} 139 \mathrm{mV} 75 \mathrm{mV} 143 \mathrm{mV}$

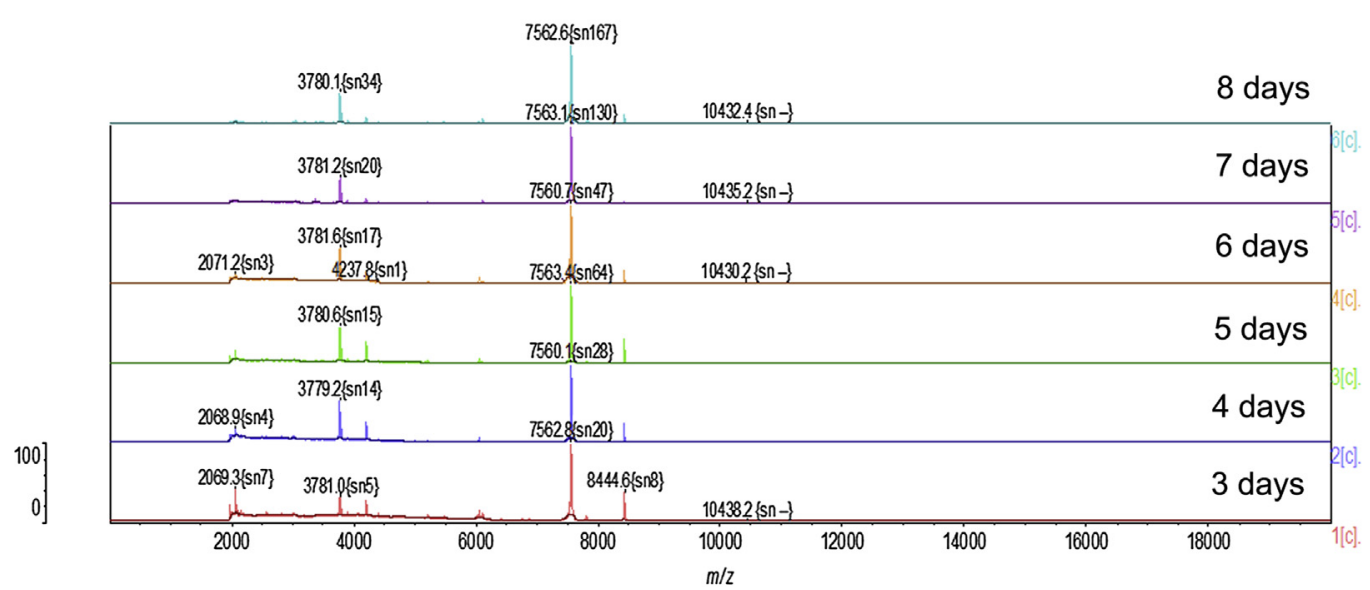

B

Shimadzu Biotech Axima LNR 2.8.6.20100624

\%lint. $\quad 99 \mathrm{mV} 1915 \mathrm{mV} 0.7 \mathrm{mV} 0.4 \mathrm{mV} 18 \mathrm{mV}$

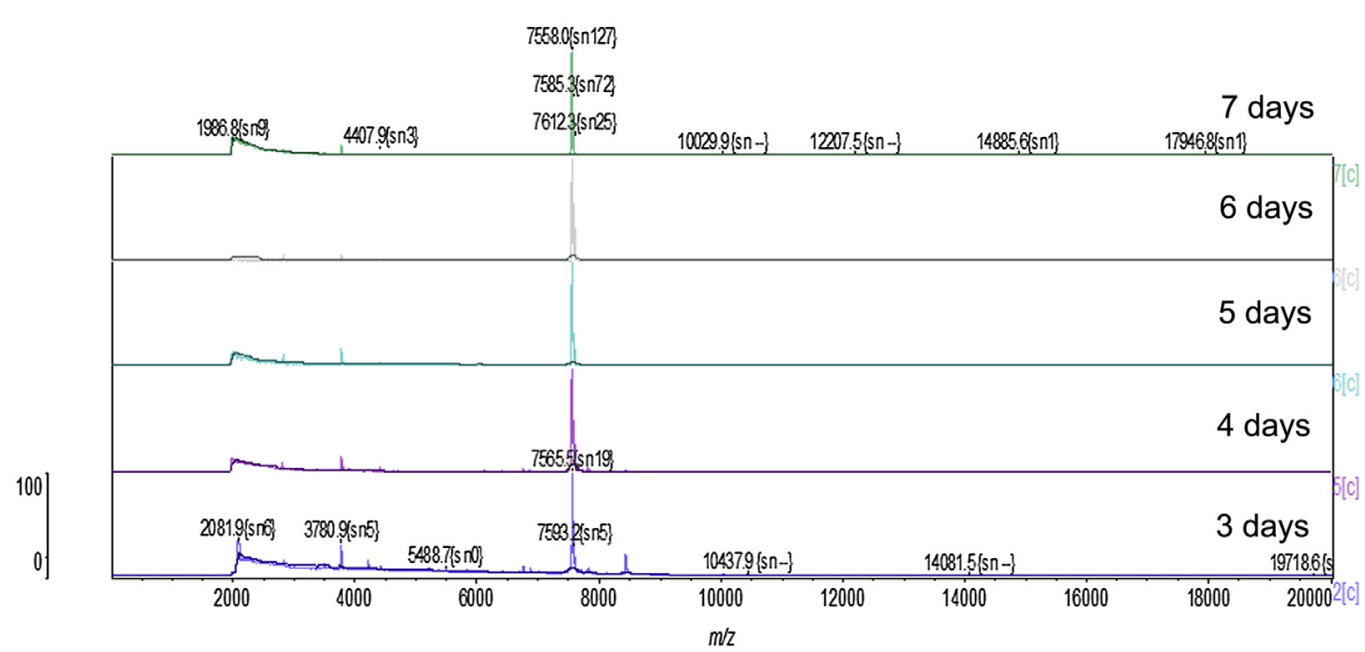

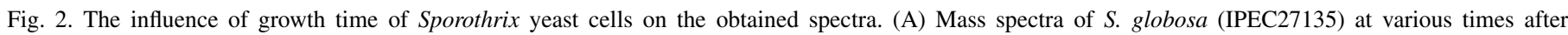

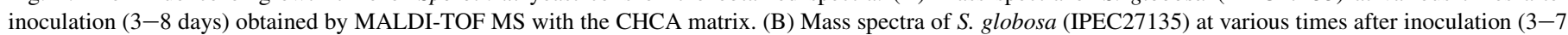
days) obtained by MALDI-TOF MS with the DHB matrix. The other tested species showed similar results.

concentrations of acids, zymolyase, protein extraction reagent, ultrasonication, glass beads and corona plasma discharge [39], have been applied as a pretreatment step. The best spectra for Sporothrix spp. were obtained when the intact yeast cells were exposed to a $25 \%$ formic acid solution and sonicated for $15 \mathrm{~min}$ at room temperature before mass spectrometric analysis.

Previous studies showed variability in mass fingerprints in the MALDI-TOF MS methodology for yeasts and filamentous fungi under different culture conditions; this variability was more pronounced among the filamentous fungi $[19,41]$. These data encouraged selection of the yeast Sporothrix phase for standardization of our MALDI-TOF MS technique. Another important factor was the higher quality of spectra obtained from yeasts with a short growth time; indeed, 3 days in the CHCA matrix led to correct identification of all species of the Sporothrix complex (Fig. 4). These results are encouraging for future use of the present protocol in direct diagnosis of clinical samples, since the yeast fungal form is the one that is present in clinical materials.

In this study, two culture media were tested for yeast growth, BHI and YEPD; we obtained better fungal growth and quality of the spectra in BHI, and absence of spectra with minimum quality in YEPD medium. These data confirmed that culture conditions may interfere with Sporothrix mass fingerprint profiles.

In conclusion, MALDI-TOF MS was able to correctly identify all clinical and environmental Sporothrix isolates using standard protocol developed in this study. MALDI-TOF MS data agreed $100 \%$ with the DNA-based method, i.e. partial sequencing of the CAL gene. This proposed identification technique is simple, reliable and accurate; it will reduce the time needed for Sporothrix identification, permitting a speed-up in epidemiologic and diagnostic studies in mycology laboratories. 


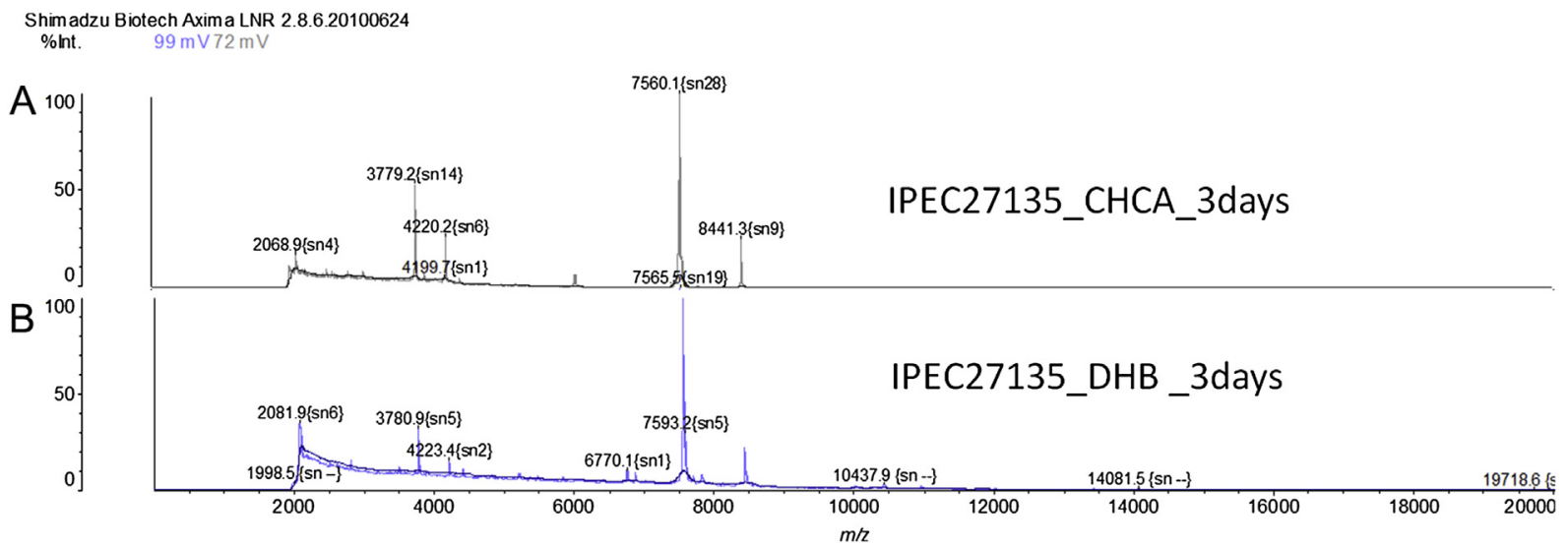

Fig. 3. Comparison between the two commonly used matrix compounds 2,5-dihydroxybenzoic acid (DHB) and $\alpha$-cyano-4-hydroxycinnamic acid (CHCA). MALDI spectra of $S$. globosa IPEC27135 were acquired in the presence of CHCA (A) and DHB (B). When CHCA was employed as the matrix, MALDI-TOF MS produced more useful mass signatures, with CHCA selected as the optimal matrix in mass signature acquisitions of all species of the Sporothrix complex.

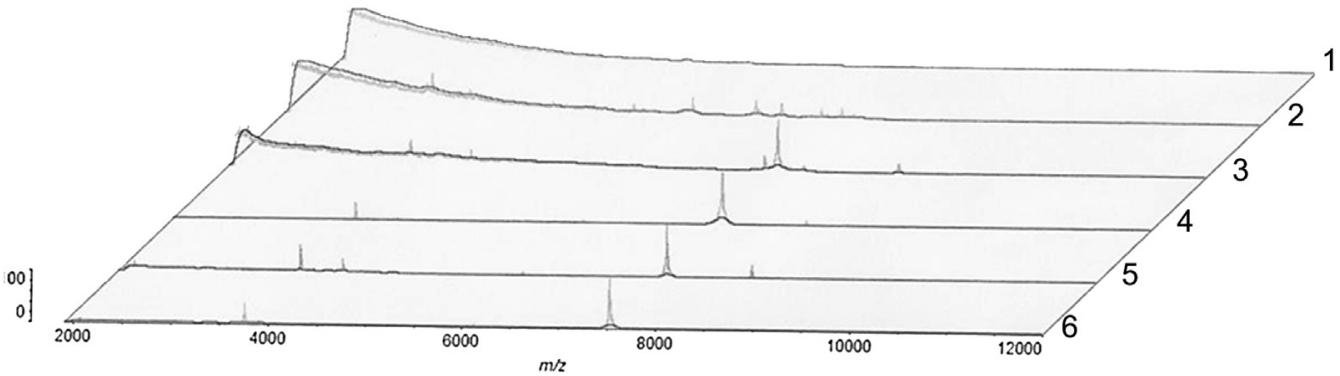

Fig. 4. Characteristic mass spectra of reference strains of the Sporothrix complex obtained by MALDI-TOF MS with the CHCA matrix. (1) S. luriei CBS937.72, (2) S. pallida SPA8, (3) S. mexicana MUM11.02, (4) S. schenckii IPEC27722, (5) S. globosa IPEC27135 and (6) S. brasiliensis CBS120339/IPEC16490.

\section{Conflict of interest}

The authors declare no conflict of interest.

\section{Acknowledgments}

The authors wish to thank the following international researchers for generously contributing strains to this study: Conchita Torrielo (EH194, EH252, EH253); Myrtha Arango (04015, 11029, 010221, 10036, 03017, 03022, 12013, 03003, 14879); and Masako Kawasaki (KMU975). Financial support was provided by FAPERJ/Rio de Janeiro, Brazil (grant proc. E-26/110.619/ 2012) and PAPES VI-Fiocruz/CNPq (Proc. 407693/2012-2) R. M. Z-O. is supported in part by CNPq 304976/2013-0 and FAPERJ E-26/103.157/2011. M. M. E. O. was supported by a grant from CAPES 2445/11-5 and PNPD/CAPES-Fiocruz/Pesquisa Clínica em Doenças Infecciosas. M. M. E. O., C. S. and N. L. thank the FCT Strategic Project PEst-OE/EQB/LA0023/2013 and BioHealth-Biotechnology and Bioengineering approaches to improve health quality, Ref. NORTE-07-0124-FEDER-000027" co-funded by the Programa Operacional Regional do Norte (ON.2 - O Novo Norte), QREN, FEDER. Automated sequencing was done using the genomic platform/DNA sequencing platform at the Fundação Oswaldo Cruz-PDTIS/ FIOCRUZ (RPT01A), Brazil.

\section{References}

[1] Oliveira MM, Almeida-Paes R, Gutierrez-Galhardo MC, ZancopeOliveira RM. Molecular identification of the Sporothrix schenckii complex. Rev Iberoam Micol 2014 Jan-Mar;31(1):2-6.

[2] Song Y, Li SS, Zhong SX, Liu YY, Yao L, Huo SS. Report of 457 sporotrichosis cases from Jilin province, northeast China, a serious endemic region. J Eur Acad Dermatol Venereol 2013 Mar;27(3):313-8.

[3] Barros MB, Almeida-Paes R, Schubach AO. Sporothrix schenckii and Sporotrichosis. Clin Microbiol Rev 2011 Oct;24(4):633-54.

[4] Liu X, Lian C, Jin L, An L, Yang G, Lin X. Characterization of Sporothrix schenckii by random amplification of polymorphic DNA assay. Chin Med J (Engl) 2003 Feb;116(2):239-42.

[5] Gutierrez-Galhardo MC, Zancopé-Oliveira RM, Valle AC, AlmeidaPaes R, Tavares PMS, Monzon A, et al. Molecular epidemiology and antifungal susceptibility patterns of Sporothrix schenckii isolates from a cat-transmitted epidemic of sporotrichosis in Rio de Janeiro, Brazil. Med Mycol 2008 Mar;46(2):141-51.

[6] Marimon R, Cano J, Gene J, Sutton DA, Kawasaki M, Guarro J. Sporothrix brasiliensis, S. globosa and S. mexicana, three new Sporothrix species of clinical interest. J Clin Microbiol 2007 Oct;45(10):3198-206.

[7] Liu TT, Zhang K, Zhou X. Molecular identification of Sporothrix clinical isolates in China. J Zhejiang Univ Sci B 2014 Jan;15(1):100-8.

[8] Oliveira MM, Almeida-Paes R, Muniz MM, Gutierrez-Galhardo MC, Zancope-Oliveira RM. Phenotypic and molecular identification of Sporothrix isolates from an epidemic area of sporotrichosis in Brazil. Mycopathologia 2011 Oct;172(4):257-67.

[9] Rodrigues AM, de Hoog S, de Camargo ZP. Emergence of pathogenicity in the Sporothrix schenckii complex. Med Mycol 2013 May;51(4): 405-12. 
[10] Dias NM, Oliveira MM, Santos C, Zancope-Oliveira RM, Lima N. Sporotrichosis caused by Sporothrix mexicana, Portugal. Emerg Infect Dis 2011 Oct;17(10):1975-6.

[11] Marimon R, Gene J, Cano J, Guarro J. Sporothrix luriei: a rare fungus from clinical origin. Med Mycol 2008 Sep;46(6):621-5.

[12] de Meyer EM, de Beer ZW, Summerbell RC, Moharram AM, de Hoog GS, Vismer HF, et al. Taxonomy and phylogeny of new wood- and soil-inhabiting Sporothrix species in the Ophiostoma stenoceras-Sporothrix schenckii complex. Mycologia 2008 Jul-Aug; 100(4):647-61.

[13] Romeo O, Scordino F, Criseo G. New insight into molecular phylogeny and epidemiology of Sporothrix schenckii species complex based on calmodulin-encoding gene analysis of Italian isolates. Mycopathologia 2011 Sep;172(3):179-86.

[14] Morrison AS, Lockhart SR, Bromley JG, Kim JY, Burd EM. An environmental Sporothrix as a cause of corneal ulcer. Med Mycol Case Rep 2013 Apr 10;2:88-90.

[15] Oliveira MM, Sampaio P, Almeida-Paes R, Pais C, GutierrezGalhardo MC, Zancope-Oliveira RM. Rapid identification of Sporothrix species by T3B fingerprinting. J Clin Microbiol 2012 Jun;50(6):2159-62.

[16] Rodrigues AM, de Hoog GS, de Camargo ZP. Genotyping species of the Sporothrix schenckii complex by PCR-RFLP of calmodulin. Diagn Microbiol Infect Dis 2014 Apr;78(4):383-7.

[17] Passarini MR, Santos C, Lima N, Berlinck RG, Sette LD. Filamentous fungi from the Atlantic marine sponge Dragmacidon reticulatum. Arch Microbiol 2013 Feb;195(2):99-111.

[18] Pereira L, Dias N, Santos C, Lima N. The use of MALDI-TOF ICMS as an alternative tool for Trichophyton rubrum identification and typing. Enferm Infecc Microbiol Clin 2013 Jan;32(1):11-7.

[19] Santos C, Lima N, Sampaio P, Pais C. Matrix-assisted laser desorption/ ionization time-of-flight intact cell mass spectrometry to detect emerging pathogenic Candida species. Diagn Microbiol Infect Dis 2011 Nov; 71(3):304-8.

[20] Santos C, Paterson RR, Venancio A, Lima N. Filamentous fungal characterizations by matrix-assisted laser desorption/ionization time-of-flight mass spectrometry. J Appl Microbiol 2010 Feb;108(2):375-85.

[21] Croxatto A, Prod'hom G, Greub G. Applications of MALDI-TOF mass spectrometry in clinical diagnostic microbiology. FEMS Microbiol Rev 2012 Mar;36(2):380-407.

[22] Lima-Neto R, Santos C, Lima N, Sampaio P, Pais C, Neves RP. Application of MALDI-TOF MS for requalification of a Candida clinical isolates culture collection. Braz J Microbiol 2014;45(2):515-22.

[23] Gautier M, Ranque S, Normand AC, Becker P, Packeu A, Cassagne C, et al. Matrix-assisted laser desorption ionization time-of-flight mass spectrometry: revolutionizing clinical laboratory diagnosis of mould infections. Clin Microbiol Infect 2014 Dec;20;12:1366-71.

[24] Qian J, Cutler JE, Cole RB, Cai Y. MALDI-TOF mass signatures for differentiation of yeast species, strain grouping and monitoring of morphogenesis markers. Anal Bioanal Chem 2008 Oct;392(3):439-49.

[25] De Carolis E, Posteraro B, Lass-Florl C, Vella A, Florio AR, Torelli R, et al. Species identification of Aspergillus, Fusarium and Mucorales with direct surface analysis by matrix-assisted laser desorption ionization time-of-flight mass spectrometry. Clin Microbiol Infect 2011 May;18(5):475-84.

[26] de Respinis S, Tonolla M, Pranghofer S, Petrini L, Petrini O, Bosshard PP. Identification of dermatophytes by matrix-assisted laser desorption/ionization time-of-flight mass spectrometry. Med Mycol 2012 Jul;51(5):514-21.

[27] Dong H, Marchetti-Deschmann M, Allmaier G. Characterization of ontarget generated tryptic peptides from Giberella zeae conidia spore proteins by means of matrix-assisted laser desorption/ionization mass spectrometry. Mol Cell Probes 2013 Apr-Jun;28(2-3):91-8.

[28] Firacative C, Trilles L, Meyer W. MALDI-TOF MS enables the rapid identification of the major molecular types within the Cryptococcus neoformans/C. gattii species complex. PLoS One 2012;7(5):e37566.

[29] Packeu A, Hendrickx M, Beguin H, Martiny D, Vandenberg O, Detandt M. Identification of the Trichophyton mentagrophytes complex species using MALDI-TOF mass spectrometry. Med Mycol 2013 Aug;51(6):580-5.

[30] Ranque S, Normand AC, Cassagne C, Murat JB, Bourgeois N, Dalle F et al. MALDI-TOF mass spectrometry identification of filamentous fungi in the clinical laboratory. Mycoses 2014 Mar;57(3):135-40.

[31] Sitterle E, Giraud S, Leto J, Bouchara JP, Rougeron A, Morio F, et al. Matrix-assisted laser desorption ionization-time of flight mass spectrometry for fast and accurate identification of Pseudallescheria/Scedosporium species. Clin Microbiol Infect 2014 Sep;20(9):929-35.

[32] Oliveira MME, Almeida-Paes R, Muniz MM, Barros MBL, GutierrezGalhardo MC, Zancope-Oliveira RM. Sporotrichosis caused by Sporothrix globosa in Rio de Janeiro, Brazil: case report. Mycopathologia 2010 May; 169(5):359-63.

[33] Suarez S, Ferroni A, Lotz A, Jolley KA, Guerin P, Leto J, et al. Ribosomal proteins as biomarkers for bacterial identification by mass spectrometry in the clinical microbiology laboratory. J Microbiol Methods 2013 Sep;94(3):390-6.

[34] Jolley KA, Bliss CM, Bennett JS, Bratcher HB, Brehony C, Colles FM, et al. Ribosomal multilocus sequence typing: universal characterization of bacteria from domain to strain. Microbiology 2012 Apr;158(Pt 4):1005-15.

[35] Roberts E, Sethi A, Montoya J, Woese CR, Luthey-Schulten Z. Molecular signatures of ribosomal evolution. Proc Natl Acad Sci U S A 2008 Sep 16;105(37):13953-8.

[36] Bennett JS, Watkins ER, Jolley KA, Harrison OB, Maiden MC. Identifying Neisseria species by use of the 50S ribosomal protein L6 (rplF) gene. J Clin Microbiol 2014 May;52(5):1375-81.

[37] Xiao D, Zhang H, He L, Peng X, Wang Y, Xue G, et al. High natural variability bacteria identification and typing: Helicobacter pylori analysis based on peptide mass fingerprinting. J Proteomics 2014 Feb 26:98:112-22.

[38] Cassagne C, Cella AL, Suchon P, Normand AC, Ranque S, Piarroux R. Evaluation of four pretreatment procedures for MALDI-TOF MS yeast identification in the routine clinical laboratory. Med Mycol 2013 May;51(4):371-7.

[39] Amiri-Eliasi B, Fenselau C. Characterization of protein biomarkers desorbed by MALDI from whole fungal cells. Anal Chem 2001 Nov 1;73(21):5228-31.

[40] Liu H, Du Z, Wang J, Yang R. Universal sample preparation method for characterization of bacteria by matrix-assisted laser desorption ionization-time of flight mass spectrometry. Appl Environ Microbiol 2007 Mar;73(6):1899-907.

[41] Erhard M, Hipler UC, Burmester A, Brakhage AA, Wostemeyer J. Identification of dermatophyte species causing onychomycosis and tinea pedis by MALDI-TOF mass spectrometry. Exp Dermatol 2008 Apr;17(4):356-61. 